\title{
PLUMPTON CORRESPONDENCE.
}

A SERIES OF LETTERS,

CHIEFLY DOMESTICK,

WRITTEN IN THE REIGNS OF

EDWARD IV. RICHARD III. HENRY VII. AND HENRY VIII.

EDITED BY THOMAS STAPLETON, ESQ. F.S.A. FROM

SIR EDWARD PLUMPTON'S BOOK OF LETTERS:

WITH

NOTICES HISTORICAL AND BIOGRAPHICAL OF THE FAMILY OP PLUMPTON, OF PLUMPTON, COM. EBOR.

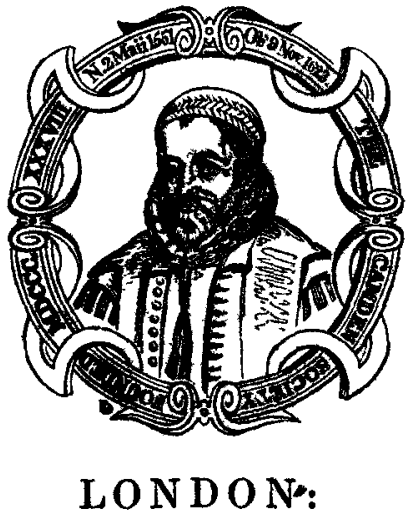

PRINTED FOR THE CAMDEN SOCIETY, BY JOHN BOWYER NICHOLS AND SON, PARLIAMENT STREET. M.DCCC.XXX.IX. 


\section{COUNCIL}

OF

THE CAMDEN SOCIETY.

\section{President,}

THE RIGHT HON. LORD FRANCIS EGERTON.

THOMAS AMYOT, ESQ. F.R.S. Treas. S.A.

JOHN BRUCE, ESQ. F.S.A. Treasurer.

JOHN PAYNE COLLIER, ESQ. F.S.A.

C. PURTON COOPER, ESQ. Q.C., D.C.L., F.R.S., F.S.A.

'T. CROFTON CROKER, ESQ. F.S.A. M.R.I.A.

REV. JOSEPH HUNTER, F.S.A.

SIR FREDERICK MADDEN, K.H., F.R.S., F.S.A.

SIR THOMAS PHILLIPPS, BART., F.R S., F.S.A.

THOMAS STAPLETON, ESQ. F.S.A.

EDGAR TAYLOR, ESQ. F.S.A.

WILLIAM JOHN THOMS, ESQ. F.S.A. Acting Sec.

THOMAS WRIGHT, ESQ. M.A., F.S.A. 